\title{
REPEATED ANTERIOR CRUCIATE LIGAMENT RUPTURES DEPENDENCE ON GENDER, THE MOST COMMON TYPES OF INJURIES AND TRANSPLANTS USED FOR LIGAMENT RECONSTRUCTION
}

\author{
Rimtautas Gudas ${ }^{1,2}$, Eimantas Spitrys ${ }^{3}$ \\ ${ }^{1}$ Lithuanian University of Health Sciences Medicine academy Orthopedics-Traumatology clinic, \\ ${ }^{2}$ Lithuanian University of Health Sciences Medicine academy Sports Institute, \\ ${ }^{3}$ Lithuanian University of Health Sciences Medicine academy
}

Key words: anterior cruciate ligament injuries, anterior cruciate ligament repeated rupture, repeated anterior cruciate ligament injuries dependence on gender, transplants and their diameters difference used for repeated anterior cruciate ligament rupture reconstruction

\begin{abstract}
Summary
The anterior cruciate ligament (ACL) is important and the primary stabilizing structure of the knee. ACL is the most commonly injured knee ligament and can cause significant morbidity in all age groups. During this study we are going to analyze what type of traumas most commonly causes primary and repeated anterior cruciate ligament injuries, what transplants and their diameters were used during reconstruction operations and repeated ACL injuries depending on gender and knee side.

Results. The average age of analyzed patients was $33.3 \pm 13.10$. Distribution of ruptures between genders was: women $23.3 \%$, men $-76.7 \%$ of cases. It was established that men had ACL repeated rupture significantly more often than women $(p<0.05)$. Left side ruptures were $43.7 \%$ of the cases and right $56.3 \%$. It was established that the injury sides distribution difference was not statistically significant $(\mathrm{p}>0.05)$. Initial ACL rupture causes $91.3 \%$ of the cases was non-contact traumas and $8.7 \%$ was contact traumas. Repeated ACL rupture causes $85.4 \%$ was non-contact traumas and $14.6 \%$ was contact traumas. Statistically significant difference was found $(\mathrm{p}<0.05)$, noncontact injuries were more frequent in both initial and repeated ruptures. Primary ACL reconstructive surgery was done $22.3 \%$ of the cases with proprium patella ligament transplant and $77.7 \%$ of the cases with semitendinosus-gracilis tendon transplant. Revision surgery was done $72.8 \%$ of the cases with pro-
\end{abstract}

prium patella ligament transplant, $20.4 \%$ semitendinosus-gracilis tendon transplant and $6.8 \%$ of the cases other types of transplants were used for reconstruction. It was established that primary ligament reconstructive surgery was performed statistically significantly more frequently with semitendinosusgracilis tendon $(p<0.05)$ and revision surgery with proprium patella ligament $(\mathrm{p}<0.05)$. Average transplants diameters used during primary ACL reconstructive surgery: semitendinosus - gracilis tendon $7.98 \pm 0.62 \mathrm{~mm}$, proprium patella ligament $8.34 \pm 1.17$ $\mathrm{mm}$. Transplants average diameters used during revision surgery: semitendinosus-gracilis tendon 8.78 $\pm 1.27 \mathrm{~mm}$, proprium patella ligament $10.11 \pm 0.85$ $\mathrm{mm}$, other transplants $9.42 \pm 0.78 \mathrm{~mm}$. The average difference of transplants diameters between the primary and revision reconstructive surgeries was 1.73 $\pm 1.39 \mathrm{~mm}$. Diameters difference between men and women was compared and no statistically significant differences were found ( $>00.05$ ).

Conclusions. Repeated ACL rupture was more common among men. Primary and secondary ACL ruptures happened more often during non-contact injury. No statistically significant difference between the sides of injury was found, both left and right violations occur at the same rate. Most commonly used transplant for the first ACL reconstruction was semitendinosus - gracilis tendon, for revision - patella proprium ligament transplant. During revision surgeries average $1.73 \mathrm{~mm}$ thicker transplants were used compared to primary reconstruction surgery.

\section{Introduction}

The anterior cruciate ligament (ACL) is important and the primary stabilizing structure of the knee. [1] [2] ACL is the most commonly injured knee ligament and can cause si- 
1 table. Distribution of anterior cruciate ligament injuries between genders

Abbreviations: $A C L$ - anterior cruciate ligament

\begin{tabular}{|l|l|l|}
\hline & Men & Women \\
\hline Repeated ACL Injuries & $76.7 \%$ & $23.3 \%$ \\
\hline & \multicolumn{2}{|c|}{$p<0.05$} \\
\hline
\end{tabular}

2 table. Types of traumas

\begin{tabular}{|l|l|l|}
\hline & Non-contact & Contact \\
\hline Initial trauma & $91.3 \%$ & $8.7 \%$ \\
\hline Repeated trauma & $85.4 \%$ & $14.6 \%$ \\
\hline & & $\mathrm{p}<0.05$ \\
\hline
\end{tabular}

3 table. Distribution of anterior cruciate ligament injuries between sides of the knee

Abbreviations: $A C L$ - anterior cruciate ligament

\begin{tabular}{|l|l|l|}
\hline & Left side & Right side \\
\hline Repeated ACL Injuries & $43.7 \%$ & $56.3 \%$ \\
\hline & \multicolumn{2}{|c|}{$\mathrm{p}>0.05$} \\
\hline
\end{tabular}

gnificant morbidity in all age groups. [2] Women experience ACL tears up to nine times more often than men. Most ACL tears occur from non-contact injuries. [1] [2] [4] Patients who sustained a contact injury have a high risk for an associated major lateral meniscus tear. [3] Multiple factors can increase the risk of ACL rupture such as increased general joint laxity, increased quadriceps angle, increased posterior tibial slope, decreased notch width, smaller ACL crosssectional area, hormonal factors, and the tendency to land with their knees in inadequate flexion and in a position of valgus and external rotation. [4] Early recognition of these risk factors may help in the prevention of injury. [2] During this study we are going to analyze what type of traumas most commonly causes primary and repeated anterior cruciate ligament injuries, what transplants and their diameters were used during reconstruction operations and repeated ACL injuries dependence on gender and knee side.

Purpose: select and analyze patients who had revision surgery because of repeated knee anterior cruciate ligament injuries and their dependence on gender, most commonly used transplants and their diameters, their differences between primary and revision surgery. Find out whether there is a statistically significant difference between the frequency of repeated injuries and the side of the knee.

\section{Methods}

During this study a retrospective analysis of repeated anterior cruciate ligament rupture reconstructive operations performed at LUHS Kaunas Clinics hospital Orthopedics - Traumatology department was done using computer databases and surgeries logbook data. Patients with repeated cruciate knee ligament injuries were selected, data from pa-
4 table. Transplant types used for ACL reconstruction Abbreviations: $A C L$ - anterior cruciate ligament, $P R$ - primary reconstruction, $P P L$ - proprium patella ligament, SGT-semitendinosus gracilis tendon

\begin{tabular}{|l|l|l|l|}
\hline & PPL & SGT & Other \\
\hline PR & $22.3 \%$ & $77.7 \%$ & $0.0 \%$ \\
\hline Revision & $72.8 \%$ & $20.4 \%$ & $6.8 \%$ \\
\hline & $\mathrm{p}<0.05$ & $\mathrm{p}<0.05$ & \\
\hline
\end{tabular}

5 table. Transplants diameters and their average difference used for ACL reconstruction

Abbreviations: $A C L$ - anterior cruciate ligament, $P R$ - primary reconstruction, $P P L$ - proprium patella ligament, $S G T$ - semitendinosus gracilis tendon, AD - average difference

\begin{tabular}{|l|l|l|l|}
\hline & PPL & SGT & Other transplants \\
\hline PR & $8.34 \pm 1.17 \mathrm{~mm}$ & $7.98 \pm 0.62 \mathrm{~mm}$ & \\
\hline Revision & $10.11 \pm 0.85 \mathrm{~mm}$ & $8.78 \pm 1.27 \mathrm{~mm}$ & $9,42 \pm 0,78 \mathrm{~mm}$ \\
\hline AD & \multicolumn{3}{|c|}{$1.73 \pm 1.39 \mathrm{~mm}$} \\
\hline
\end{tabular}

tients medical records and collected data from other hospitals about primary cruciate knee ligament surgery was used. 103 anterior cruciate ligament revision surgery cases were analyzed, all these patients had repeated anterior cruciate ligament ruptures. Patients were divided into two groups according to gender and injury side, left or right. Analyzed what injury types contact or non-contact occurs more frequently, what types of ligament transplants were used during primary and revision surgery and diameter difference of transplants used during primary and revision surgeries between men and women. Calculated average age of the patients. Statistical analysis was performed using a standard statistical package SPSS for Windows 21.0. Chi square and Mann Whitney statistical criteria were used for analysis, the chosen level of significance $p<0.05$. Mann Whitney criterion was used to find out whether is a difference in transplants diameters between men and women. Chi square criterion was used to find out whether repeated ligament injuries were more frequent for men or women, which side was damaged more frequently, left or right, which type of transplant was used most commonly and what types of traumas caused anterior cruciate ligament rupture most commonly.

\section{Results}

The average age of analyzed patients was $33.3 \pm 13.10$. Table 1 shows the distribution of ACL ruptures between genders, $\mathrm{p}$-value $<0.05$ shows that men had repeated ACL rupture more often than women. Table 2 shows types of traumas which caused primary and repeated ACL ruptures. Statistically significant difference was found, $p$-value $<0.05$, non-contact injuries were more frequent during primary and repeated ruptures. Table 3 shows ACL ruptures distribution between sides, it was established that the injury sides distribution difference was not statistically significant $(p>0.05)$. It 
was established that primary ligament reconstructive surgery was performed statistically significantly more frequently with semitendinosus-gracilis tendon and revision surgery with proprium patella ligament (table 4). Average transplants diameters and their difference used during primary cruciate ligament reconstructive surgery are shown in table 5. Diameters difference between men and women was compared and no statistically significant differences were found $(\mathrm{p}>0.05)$.

\section{Conclusions}

Repeated ACL rupture was more common among men. Primary and secondary ACL ruptures happened more often during non-contact than contact injury. No statistically significant difference between the sides of injury was found, both left and right violations occur at the same rate. Most commonly used transplant for the first ACL reconstruction was semitendinosus - gracilis tendon After first transplant rupture, for revision, most commonly used transplant was patella proprium ligament. During revision surgeries average $1.73 \mathrm{~mm}$ thicker transplants were used compared to primary reconstruction surgery.

\section{References}

1. Cimino F, Volk BS, Setter D. Anterior cruciate ligament injury: diagnosis, management, and prevention. Am Fam Physician 2010; 82:917-922.

2. Mahajan PS, Chandra P, Negi VC, Jayaram AP, Hussein SA. Smaller anterior cruciate ligament diameter is a predictor of subjects prone to ligament injuries: an ultrasound study. Biomed Res Int 2015.

3. Feucht MJ, Bigdon S, Bode G, Salzmann GM, Dovi-Akue D, Südkamp NP, Niemeyer P. Associated tears of the lateral meniscus in anterior cruciate ligament injuries: risk factors for different tear patterns. J Orthop Surg Res 2015; 10(1):34.

4. Tan SH, Lau BP, Khin LW, Lingaraj K. The importance of patient sex in the outcomes of anterior cruciate ligament reconstructions: a systematic review and meta-analysis. Am J Sports Med 2015.

\section{PAKARTOTINŲ PRIEKINIO KRYŽMINIO RAIŠČČO PLYŠIMŲ PRIKLAUSOMYBE் NUO LYTIES, DAŽNIAUSI TRAUMU TIPAI IR TRANSPLANTATAI NAUDOTI RAIŠČIU REKONSTRUKCIJAI \\ R. Gudas, E. Spitrys}

Raktažodžiai: priekinio kryžminio raiščio pažeidimai, priekinio kryžminio raiščio pakartotiniai plyšimai, pakartotinų priekinio kryžminio raiščio plyšimų priklausomybè nuo lyties, transplantatai ir jų diametrų skirtumai naudoti priekinio kryžminio raiščio rekonstrukcijai

Santrauka

Priekinis kryžminis raištis (PKR) yra svarbi ir viena pagrin- dinių kelio sąnario stabilizavimo struktūrų. PKR yra dažniausiai pažeidžiamas kelio raištis ir gali sukelti didelị sergamumą visose amžiaus grupèse. Šio tyrimo metu nustatoma, kokios rūšies traumos dažniausiai sukelia pirminius ir pakartotinius priekinių kryžminių raiščių plyšimus, kokie transplantatai ir jų diametrai dažniausiai buvo naudojami ir pakartotinų PKR plyšimų priklausomybę nuo lyties ir kelio pusès.

Rezultatai. Vidutinis tirtų pacientų amžius buvo $33,3 \pm 13,10$ metai. Plyšimų pasiskirstymas tarp lyčių buvo: moterims $23,3 \%$, vyrams $-76,7 \%$ plyšimų atvejų. Buvo nustatyta, kad vyrai dažniau nei moterys turejjo priekinio kryžminio raiščio pakartotinius plyšimus $(\mathrm{p}<0.05)$. Kairèje kelio pusèje PKR plyšimas buvo $43,7 \%$, dešinèje $-56,3 \%$ atvejų. Statistiškai reikšmingo skirtumo tarp pažeidimo pusių nebuvo nustatyta $(\mathrm{p}>0,05)$. Nustatyta, kad pirminį PKR plyšimą 91,3\% atvejų sukelia nekontaktinès traumos ir $8,7 \%$ atvejų kontaktinès. Pakartotinius PKR plyšimus $85,4 \%$ atvejų sukèlè nekontaktinès traumos ir $14,6 \%$ atvejų kontaktinès. Nustatytas statistiškai reikšmingas skirtumas $(\mathrm{p}<0,05)$ nekontaktinio tipo traumos dažniau sukẻle PKR plyšimus. Pirminio PKR plyšimo rekonstrukcinè operacija $22,3 \%$ atvejų buvo atlikta su savojo girnelès raiščio transplantatu, $77,7 \%$ atvejų su semitendinosus - gracilis sausgyslès transplantu. Revizinè operacija 72,8\% atvejų atlikta su girnelès savojo raiščio transplantu, $20,4 \%$ atvejų su semitendinosus - gracilis sausgyslès transplantu ir 6,8\% atvejų rekonstrukcijai buvo naudojami kitų tipų transplantatai. Buvo nustatyta, kad pirminè PKR rekonstrukciné operacija buvo atlikta statistiškai reikšmingai dažniau su semitendinosus - gracilis sausgyslių transplantatu $(\mathrm{p}<0,05)$, o revizinè operacija su savuoju girnelès raiščio transplantatu $(p<0,05)$. Vidutinis naudotų transplantatų skersmuo pirminés PKR rekonstrukcijos metu: semitendinosus - gracilis sausgysliu transplantatu 7,98 $\pm 0,62 \mathrm{~mm}$, savavojo girnelè raiščio transplantatų $8,34 \pm 1,17 \mathrm{~mm}$. Revizinių operacijų metu naudotu transplantatų vidutinis skersmuo: semitendinosus - gracilis sausgyslių transplantatų $8,78 \pm 1,27 \mathrm{~mm}$, savojo girnelès raiščio transplantatų $10,11 \pm 0,85 \mathrm{~mm}$, kitų rekonstrukcijai naudotų transplantatų $9,42 \pm 0,78 \mathrm{~mm}$. Vidutinis transplantatų skersmenų skirtumas tarp pirminių ir revizinių PKR rekonstrukcinių

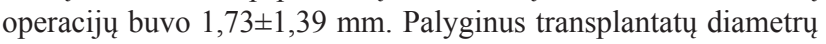
skirtumus tarp vyrų ir moterų statistiškai reikšmingų skirtumų nenustatyta $(\mathrm{p}>0,05)$.

Išvados. Pakartotiniai priekinio kryžminio raiščio plyšimai labiau paplitę tarp vyrų . Pirminiai ir pakartotiniai priekinio kryžminio raiščio plyšimai dažniausiai ịvyko nekontaktinès traumos metu. Statistiškai reikšmingo skirtumo tarp pažeidimų pusių nebuvo nustatyta. Dažniausiai naudojami transplantatai pirminès priekinio kryžminio raiščio rekonstrukcijos metu buvo semitendinosus - gracilis sausgyslès, revizijos - girnelès savojo raiščio transplantatas. Revizinių operacijų metu lyginant su pirminėmis priekinio kryžminio raiščio rekonstruktinemis operacijomis vidutiniškai buvo naudota $1,73 \mathrm{~mm}$ storesni transplantatai.

Adresas susirašinèti: eimantas.spitrys@gmail.com

Gauta 2015-05-04 